\title{
Clinical features and diagnosis of aspirin induced asthma
}

Andrzej Szczeklik, Ewa Niżankowska

Although for therapeutic reasons it has become convenient to consider asthma as a single disease entity, this clearly is not the case, with many variants occurring. From a clinical standpoint, a minimal subdivision includes atopic asthma, cough variant asthma, brittle asthma, intrinsic asthma, occupational nonIgE dependent asthma, and aspirin intolerant asthma (AIA). ${ }^{1}$ This last variant constitutes a clearcut clinical syndrome. It is a remarkable model for investigating mechanisms that operate in asthma, rhinitis, and nasal polyposis. The recent introduction of anti-leukotriene drugs has amplified interest in this syndrome.

\section{Definition, prevalence and clinical presentation}

AIA is an aggressive mucosal inflammatory disease combined with precipitation of asthma and rhinitis attacks which occurs after ingestion of aspirin and most non-steroidal antiinflammatory drugs (NSAID). ${ }^{2}$ Aspirin intolerance is underdiagnosed within the asthmatic population. Based on patients' history alone, the incidence of aspirin sensitivity in adult asthmatics is $3-5 \%$, but this rises to $19 \%$ of consecutive adult asthmatic patients challenged with oral aspirin. Even in adult asthmatics with no history of aspirin intolerance, $9 \%$ show sensitivity to oral aspirin challenge and in those with rhinosinusitis the figure rises to $34 \% .^{3}$ The reasons for underreporting of aspirin sensitivity may include the deliberate avoidance of NSAIDs by asthmatic patients who are aware of adverse reactions, or a lack of recognition by patients of mild NSAID induced reactions because of their delayed onset of action. Thus, in a population of 500 patients with AIA studied in the European Network of Aspirin-Induced Asthma (AIANE), 18\% were unaware of aspirin intolerance before having aspirin provocation tests. This indicates that underdiagnosis of aspirin sensitivity may be due to the lack of routine aspirin challenge testing of asthmatic patients who do not report a positive history of aspirin sensitivity.

In most patients (women are affected 2.5 times more often than men), symptoms of rhinitis first occur during the third decade, often after a viral respiratory illness. ${ }^{2}{ }^{4}$ Over a period of months, chronic nasal congestion, anosmia, and rhinorrhoea develop. Physical examination often reveals nasal polyps. Bronchial asthma and sensitivity to aspirin develops next. After ingestion of aspirin or an NSAID, an acute asthma attack occurs within a few minutes up to three hours, usually accompanied by profuse rhinorrhoea, conjunctival infection, periorbital oedema, and sometimes a scarlet flushing of the head and neck. Aspirin is a common precipitant of life threatening attacks of asthma. In a large survey, $25 \%$ of asthmatic patients requiring emergency mechanical ventilation were found to have AIA. ${ }^{6}$

Aggressive nasal polyposis and asthma run a protracted course, despite the avoidance of aspirin and cross reacting drugs. Blood eosinophil counts are raised and eosinophils are present in the nasal mucosa and bronchial airways. Although at one time AIA was thought not to occur in atopic patients, the frequency of positive skin test responses to common aeroallergens is similar in patients with AIA and those with other types of asthma. ${ }^{78}$

By far the most accepted explanation for the pharmacological and clinical features of AIA is the cyclo-oxygenase (COX) theory ${ }^{9}$ which states that precipitation of asthmatic attacks is caused by inhibition of COX by aspirin-like drugs in the respiratory tract of the patients. The pathophysiology is characterised in part by excess production of cysteinyl leukotrienes (cys-LT). Basal excretion of cys-LT in urine is increased in AIA and increases further upon aspirin administration. ${ }^{10}$ In addition, following aspirin challenge there is a release of cys-LT into both the nasal cavity and bronchial tract. ${ }^{112}$ Leukotriene $\mathrm{C}_{4}\left(\mathrm{LTC}_{4}\right)$ synthase, the terminal enzyme for cys-LT production, is markedly overexpressed in bronchial biopsy specimens from patients with AIA. ${ }^{13}$ An allelic variant of $\mathrm{LTC}_{4}$ synthase which enhances the enzyme transcription is also associated with a more severe steroid dependent form of AIA. ${ }^{14}$

\section{Diagnosis of aspirin intolerance}

The following clues in a patient's history might give rise to the suspicion of AIA: (1) typical symptoms of aspirin induced respiratory reactions; (2) severe asthma accompanied by chronic nasal congestion and profuse rhinorrhoea; (3) frequent development of nasal polyps; and (4) sudden severe attack of asthma requiring admission to an intensive care unit. $^{25} 1516$

There is no in vitro reliable test for the diagnosis of aspirin intolerance. The diagnosis can only be established with certainty by aspirin provocation tests. ${ }^{217}$ There are four types of provocation tests, depending on the manner of aspirin administration: oral, bronchial (inhaled), nasal, and intravenous.

Oral challenge tests were introduced systematically into clinical practice in the early 1970s in Poland. ${ }^{15}$ They consisted of administration of placebo and increasing doses of aspirin during four consecutive days. The aspirin challenge test mmszczek@cyf-kr.edu.pl 
was considered positive if a fall in forced expiratory volume in one second $\left(\mathrm{FEV}_{1}\right)$ of more than $20 \%$ occurred, usually accompanied by bronchoconstriction and nasal symptoms. Oral challenge procedures were later introduced by some authors in the USA and in Europe. ${ }^{1618}$ The protocols differed between the respective clinical centres in the dosage of aspirin used, the intervals between the successive doses, and the criteria for assessing the test as positive. For instance, Stevenson et $a l^{16}$ administered placebo and increasing doses of aspirin over three consecutive days, while Dahlen and Zetterstrom ${ }^{18}$ administered increasing doses of aspirin during a one day long challenge procedure with very short intervals between the consecutive doses.

Inhalation (bronchial) tests for the diagnosis of aspirin intolerance were introduced into clinical practice by Bianco et al in 1977. ${ }^{19}$ They were based on the administration of increasing concentrations of lysine-aspirin. In the following years the inhalation challenges were also used by Schmitz-Schumann et al, ${ }^{20}$ Phillips et $a l,{ }^{21}$ and Dahlen and Zetterstrom. ${ }^{18}$ Inhalation of increasing concentrations of lysine-aspirin proved safer and quicker than the oral challenges, although the symptoms provoked were usually restricted to the airways.

For many years nasal challenge tests with histamine, methacholine, and allergens have been used for research purposes and in clinical studies. Nasal tests with lysine-aspirin have been used sporadically for the diagnosis of AIA. ${ }^{22}{ }^{23}$ We have recently developed a diagnostic nasal lysine-aspirin challenge test ${ }^{24}$ using a total dose of $16 \mathrm{mg}$ acetylsalicylic acid applied bilaterally into the inferior nasal conchae. The response is evaluated clinically and by anterior rhinomanometry. The test is highly sensitive and specific, but the negative results do not exclude possible intolerance to aspirin. The predictive value of a negative result was only $78.6 \% .^{24} \mathrm{~A}$ nasal provocation test carried out in line with our procedure is a simple, safe, and quick diagnostic method for the assessment of aspirin intolerance.

Some authors have used intravenous provocation tests with anti-inflammatory drugs. For instance, Martelli et $a l^{25}$ administered indomethacin intravenously while Taniguchi et $a l^{26}$ used lysine-aspirin intravenously.

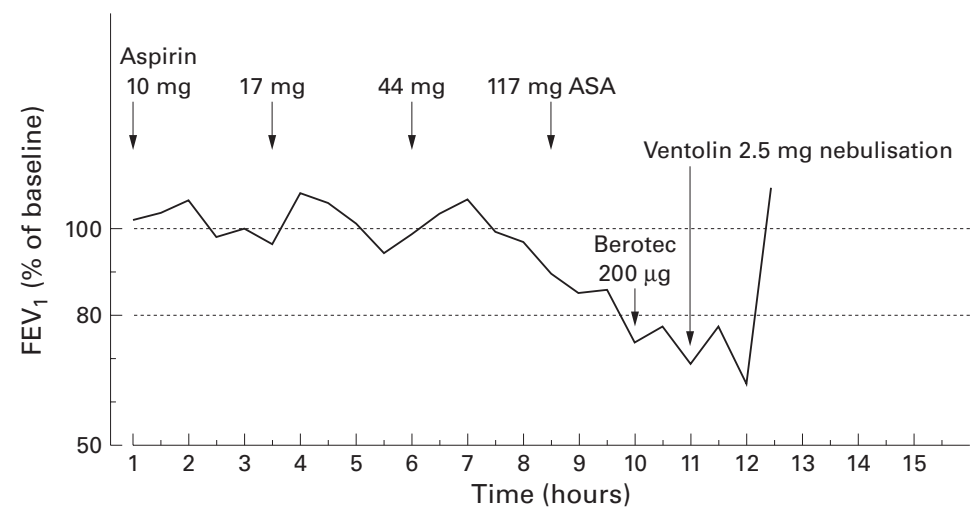

Figure 1 Example of an oral aspirin challenge test.
The protocols for both oral and bronchial tests differ between various clinical centres. We recently developed similar procedures for carrying out both oral and inhalation (bronchial) tests with aspirin. ${ }^{27}$ The cumulative doses of aspirin in the oral challenges and of lysine-aspirin in the bronchial challenges increased in geometric progression (oral cumulative dose $500 \mathrm{mg}$ aspirin, bronchial cumulative dose $182 \mathrm{mg}$ ). This method has made it possible to calculate the provocative dose of aspirin leading to a $20 \%$ fall in $\mathrm{FEV}_{1}$, both during oral and bronchial challenges $\left(\mathrm{PD}_{20}\right.$ oral and $\mathrm{PD}_{20}$ bronchial values). The oral test performed in line with our new protocol proved positive in $77.14 \%$ of patients studied, based on a $20 \%$ decrease in $\mathrm{FEV}_{1}$. When the strong extrabronchial symptoms were also included in the criteria, the test proved positive in $88.57 \%$ of patients.

Bronchial lysine-aspirin challenge led to a decrease in $\mathrm{FEV}_{1}$ of at least $20 \%$ in $60 \%$ of patients studied. In $17.17 \%$ of patients it proved positive when only the extrabronchial symptoms were considered. In some patients the inhaled test proved repeatedly negative, despite a positive oral challenge test. Both oral and bronchial tests had similar specificity, but the sensitivity of the oral test was somewhat higher. The inclusion of extrabronchial symptoms into the assessment criteria enhanced the diagnostic value of both procedures (fig 1).

Before carrying out provocation challenges, short acting $\beta$-mimetics should be stopped for eight hours, long acting $\beta$-mimetics for 24 hours before the tests, and theophylline 24-48 hours before testing. Antihistamines should be discontinued one week earlier. Oral and inhaled aspirin challenges should always be performed in patients with baseline $\mathrm{FEV}_{1}$ $>60 \%$ of the predicted value. As corticosteroid treatment can attenuate aspirin precipitated adverse reactions in patients with AIA ${ }^{28}$ we do not usually perform any provocation tests in patients treated with oral corticosteroids in doses of more than $10 \mathrm{mg}$ prednisolone daily.

\section{Conclusions}

Provocation challenges with increasing doses of aspirin or lysine-aspirin are the only reliable methods for diagnosis of aspirin intolerance. Oral challenges remain the gold standard for the diagnosis of aspirin intolerance but they may precipitate acute asthmatic reactions and therefore should be performed in specialised centres only. Bronchial challenges are safer and quicker but have somewhat less sensitivity than oral challenges. Nasal challenges are safe and may be carried out in almost all allergy centres as the routine screening procedure, even in patients with unstable asthma. It is the method of choice for confirming intolerance to aspirin when manifested only by symptoms originating in the upper respiratory tract. Patients suspected of having aspirin intolerance with negative nasal provocation tests should undergo bronchial and/or oral challenge tests with aspirin.

1 Holgate ST. Genetic and environmental interactions in allergy and asthma. F Allergy Clin Immunol 1999;104:113946. 
2 Szczeklik A, Stevenson DD. Aspirin-induced asthma: advances in pathogenesis and management. 7 Allergy Clin advances in pathogenesi

3 Sampson AP. Drug-induced asthma: NSAIDs, betablockers and ACE inhibitors. In: Holgate ST, Boushey HA Fabbri LM, eds. Difficult asthma. London: Martin Dunitz, 1999: 127-46.

4 Samter M, Beers RF. Intolerance to aspirin. Clinical studies and consideration of its pathogenesis. Ann Intern Med 1968;68:975-83.

5 Nizankowska E, Duplaga M, Bochenek G, Szczeklik A, on behalf of the AIANE Project. Clinical course of aspirininduced asthma: results of AIANE. In: Szczeklik A Gryglewski R, Vane J, eds. Eicosanoids, aspirin and asthma. New York: Marcel Dekker, 1998: 451-71.

6 Marquette $\mathrm{CH}$, Saulnier F, Leroy O, et al. Long-term prognosis for near-fatal asthma. A 6-year follow-up study of 145 asthmatic patients who underwent mechanical ventilation for near-fatal attack of asthma. Am Rev Respir Dis 190 near-fatal a $7 \begin{gathered}\text { Bochenek G, Nizankowska E, Szczeklik A. Atopy trait in } \\ \text { hypersensitivity to nonsteroidal anti-inflammatory drugs. }\end{gathered}$ hypersensitivity to nonst
Allergy 1996;51:16-23.

8 Kalyoncu AF, Karakaya G, Sahin AA, et al. Occurrence of allergic conditions in asthmatics with analgesic intolerance. Allergy 1999;54:428-35

9 Szczeklik A. The cyclooxygenase theory of aspirin-induced asthma. Eur Respir f 1990;3:588-93.

10 Nasser SMS, Lee TH. Leukotrienes in aspirin-sensitive asthma. In: Szczeklik A, Gryglewski R, Vane J, eds. Eicosanoids, aspirin and asthma. New York: Marcel Dekker, 1998 : 317-35.

11 Kowalski ML, Sliwinska-Kowalska M, Igarashi Y, et al. Nasal secretions in response to acetylsalicylic acid. F Allergy Clin Immunol 1993;91:580-98.

12 Szczeklik A, Sladek K, Dworski R, et al. Bronchial aspirin challenge causes specific eicosanoid response in aspirin sensitive asthmatics. Am $\mathcal{F}$ Respir Crit Care Med 1996;154: sensitive 1608 - 14 .

13 Cowburn AS, Sladek K, Soja J, et al. Overexpression of leukotriene C4 synthase in bronchial biopsies from patients kotriene C4 synthase in bronchial biopsies from patients with

14 Sanak M, Simon H-U, Szczeklik A. Leukotriene C4 synthase promoter polymorphism and risk of aspirininduced asthma. Lancet 1997;350:1599-600.

15 Szczeklik A, Gryglewski R, Czerniawska-Mysik G. Relationship of inhibition of prostaglandin biosynthesis by analgesics to asthma attacks in aspirin-sensitive patients. $B M \mathcal{F}$ $1975 ; 1: 67-9$
16 Stevenson DD, Simon RA. Sensitivity to aspirin and nonsteroidal anti-inflammatory drugs. In: Middleton E, Reed CE, Ellis EF, Adkinson NF, Yunginger J, eds. Allergy: principle and practice. Volume 2. St Louis, Missouri: Mosby-Year Book. 1993:1747-65.

17 Szczeklik A, Nizankowska E. Pharmacological agents in bronchial provocation tests. In: Allegra L, Braga PC, Dal Negro R, eds. Methods in asthmology. Berlin: Springer Verlag, 1993: 253-64.

18 Dahlen B, Zetterström O. Comparison of bronchial and peroral provocation with aspirin in aspirin-sensitive asthmatics. Eur Respir F 1990;3:527-34.

19 Bianco S, Robuschi M, Petrigni G. Aspirin induced tolerance in aspirin-asthma detected by a new challenge test. $\mathcal{F}$ Med Sci 1977;5:129-30.

20 Schmitz-Schumann M, Schaub E, Virchow C. Inhalative Provocation mit Lysin-Azetylsalizylsaure bei AnalgeticaAsthma-Syndrom. Prax Pneumol 1982;36:17-21.

21 Phillips GD, Foord R, Holgate ST. Inhaled lysine-aspirin as a bronchoprovocation procedure in aspirin-sensitive asthma: its repeatability, absence of a late-phase reactions, and the role of histamine. $\mathcal{F}$ Allergy Clin Immunol 1989;84: $232-41$

22 Pawlowicz A, Williams W, Davies B. Inhalation and nasal challenge in the diagnosis of aspirin-induced asthma. Allergy 1991;46:405-9.

23 Schapowal A, Schmitz-Schumann M, Szczeklik A, et al. Lysine-aspirin nasal provocation and anterior rhinomanometry for the diagnosis of aspirin-sensitive asthma. Atemw-Lungenkrkh 1990;16(Suppl 1):1-5.

24 Milewski M, Mastalerz L, Nizankowska E, et al. Nasal provocation test with lysine-aspirin for diagnosis of aspirinsensitive asthma. F Allergy Clin Immunol 1998,5:581-6.

25 Martelli NA. Bronchial and intravenous provocation tests with indomethacin in aspirin-sensitive asthmatics. Am Rev Respir Dis 1979;120:1073-9.

26 Taniguchi M, Suetsugu S, Sakakibara H, et al. Anti-viral agent, aciclovir, significantly reduces aspirinhyperresponsiveness in patients with aspirin-induced asthma. Am 7 Respir Crit Care Med 1997;155:A974.

27 Nizankowska E, Bestynska-Krypel A, Cmiel A, et al. Oral and bronchial provocation tests with aspirin for diagnosis of aspirin-induced asthma. Eur Respir f 2000;15:863-9.

28 Nizankowska E, Szczeklik A. Glucocorticosteroids attenuate aspirin-precipitated adverse reaction in aspirin-intolerant patients with asthma. Ann Allergy 1989;63:159-64. 